\title{
Design with Constructal Theory
}

\author{
A. Bejan ${ }^{1}$ and S. Lorente ${ }^{2}$ \\ ${ }^{1}$ Duke University, Department of Mechanical Engineering and Materials Science, Box 90300, \\ Durham, NC 27708-0300, USA \\ ${ }^{2}$ University of Toulouse, INSA, Laboratory of Materials and Durability of Constructions, \\ 135 avenue de Rangueil; F-31077 Toulouse, France
}

Keywords: constructal, design in nature, tree networks, shape, structure

\begin{abstract}
Constructal theory is the view that the generation of "designedness" in nature is a universal (physics) phenomenon that can be based on principle (the constructal law): "For a finite-size flow system to persist in time (to live) its configuration must change in time so that it provides greater and greater access to its currents". This principle predicts natural form across the board, from river basins to animal design, engineering and social dynamics. In this introduction to the theory we show examples of vascular designs at large and small scales and multi-objective flow configurations.
\end{abstract}

\section{The constructal law of generation of flow configuration}

In this review we draw attention to a new book [1] in which we used constructal theory to design strategies for more effective flow and transport. Constructal theory is the view that the generation of flow configuration in a universal phenomenon of all physics, which is covered by a principle (the constructal law):

"For a finite-size flow system to persist in time (to live) it must evolve such that it provides greater and greater access to the currents that flow through it" $[2,3]$.

This principle has been used to account for many features of "designedness" in nature, from the tree-shaped flows of lungs and river basins, to the scaling laws of animal design, river basin design and social dynamics. This is now a fast growing field with contributions from many sources, and with leads in many directions. This body of work has two sides. One is the use of the constructal law to predict and explain the occurrence of natural flow configurations, inanimate and animate [3-6]. The other side is the application of the constructal law as a physics principle in engineering. This philosophy of design as science is the main thread of the new book [1].

In science, the origin (genesis) of the configuration of flow systems has been overlooked. Design has been taken for granted - at best, it has been attributed to chance, inspiration, talent, and art. Our own education in the sciences is based on sketches of streams into and out of boxes, sketches that bear no relation to reality, to the position that the stream occupies in space and in time. Constructal theory and design is a proposal to change this attitude.

The benefits from thinking of design as science are great. For example, the march toward smaller dimensions (micro, nano) - the miniaturization revolution - is not about making smaller and smaller components that are to be dumped like sand into a sack. This revolution is about the "living 
sack", in which every single component is kept alive with flows that connect it to all the other components. Each component is put in the right place, like the neurons in the brain, or the alveoli in the lung, the hill slopes in the river basin, and the mid-size cities on the map. It is the configuration of these extremely numerous components that makes the "whole" perform best.

Because natural flow systems have configuration, with constructal theory we treat the emergence of flow configuration as a phenomenon that is based on a scientific principle. Constructal theory is the mental viewing that the generation of the flow structures that we see everywhere in nature (river basins, lungs, atmospheric circulation, vascularized tissues, etc.) can be reasoned based on an evolutionary principle of increase of flow access in time. That principle is the constructal law.

Geometry or drawing is not a figure that always existed and now is available to look at, or worse, to look through and take for granted. The figure is the persistent movement, struggle, contortion and mechanism by which the flow system achieves global objective under global constraints. When the flow stops, the figure becomes a flow 'fossil' (e.g., dry river bed, snowflake, animal skeleton, abandoned technology, and pyramids of Egypt).

Constructal theory has brought many researchers and educators together, from many fields (engineering, physics, biology, sociology, government) and in a new direction: to use the constructal law for better engineering and for better organization of the movement and connecting of people, goods and information [5-7]. We call this direction constructal design, and with it we seek not only better configurations but also better (faster, cheaper, direct, reliable) strategies for generating the geometry that is missing.

For example, the best configurations that connect one component with very many components are tree-shaped, and for this reason dendritic flow architectures occupy a central position in the field. Trees are flows that make connections between discrete points and continua, i.e., between one point and infinities of points, namely, between a volume and one point, an area and one point, and a curve and one point. The flow may proceed in either direction, e.g. volume-to-point and point-to-volume.

Trees are not the only class of multi-scale designs to be discovered and used. In Ref. [1] for example, we also teach how to develop multi-scale spacings that are distributed nonuniformly through a flow package, flow structures with more than one objective, and, especially, structures that must perform both flow and mechanical support functions. Along this route, we unveil designs that have more and more in common with animal design. We do all this by invoking a single principle (the constructal law), not by copying from nature.

With 'animal design' as an icon of ideality in nature, the better name for the miniaturization and globalization trends that we see emerging is vascularization. Every multi-scale solid structure that is to be cooled, heated or serviced by our fluid streams must be and will be vascularized. This means trees and spacings and solid walls, with every geometric detail sized and positioned in the right place in the available space. These will be solid-fluid structures with multiple scales that are distributed nonuniformly through the volume - so nonuniformly that the 'design' may be mistaken as random (the result of chance) by those who do not know the generating principle. This is just like in the prevailing view of animal design, where diversity is mistaken for randomness, when in fact the diversity is the fingerprint of the constructal law [8-10].

The future of our designs belongs to the vascularized, at all scales. Our 'hands' (streams, inlets, outlets) are few, but they must reach the infinity of points of the volume of material that serves us (the devices, the artefacts, i.e. the engineered extensions of the human body). Point-volume and point-area flows call for the use of tree-shaped configurations. The comparison with the 
vascularization of animal tissue (or urban design and globalization at larger scales) is another hint that to design with constructal theory is the way of the future. Our machines are moving toward animal-design configurations: distributed power generation on the landscape and on vehicles, distributed drives, distributed refrigeration, distributed computing, etc. All these distributed schemes mean trees mating with trees, i.e. vascularization [1].

\section{Two flow mechanisms are better than one}

The design feature that unites many of the natural flow configurations is that greater flow access can be achieved if there is more than one mechanism at the disposal of the currents that must flow. The tree-shaped flows of nature are an icon of this duality: organized flow (streams) along the channels coexists with disorganized flow (diffusion) across the spaces between channels. There is a balance between the two flow mechanisms, and between large channels and small channels (e.g., Section 3).

In all the river basins on earth, the time scale of seepage down the hill slope is the same as the residence time in the entire basin [11]. This balance between dissimilar flow mechanisms makes the river basin flow in the same way as the lung, where there is a balance between flow resistance along the airways and across the alveolus. The river basin and the lung maximize the flow access between one point and an infinity of points (area, volume).

The turbulent eddy is born out of the same diffusion-convection balance [2]. Consider the flow of momentum by shear, from a semi-infinite fluid of uniform speed V to the rest of the fluid, which is stationary. The shear plane becomes coated with a layer of thickness $D$ that increases in time. If

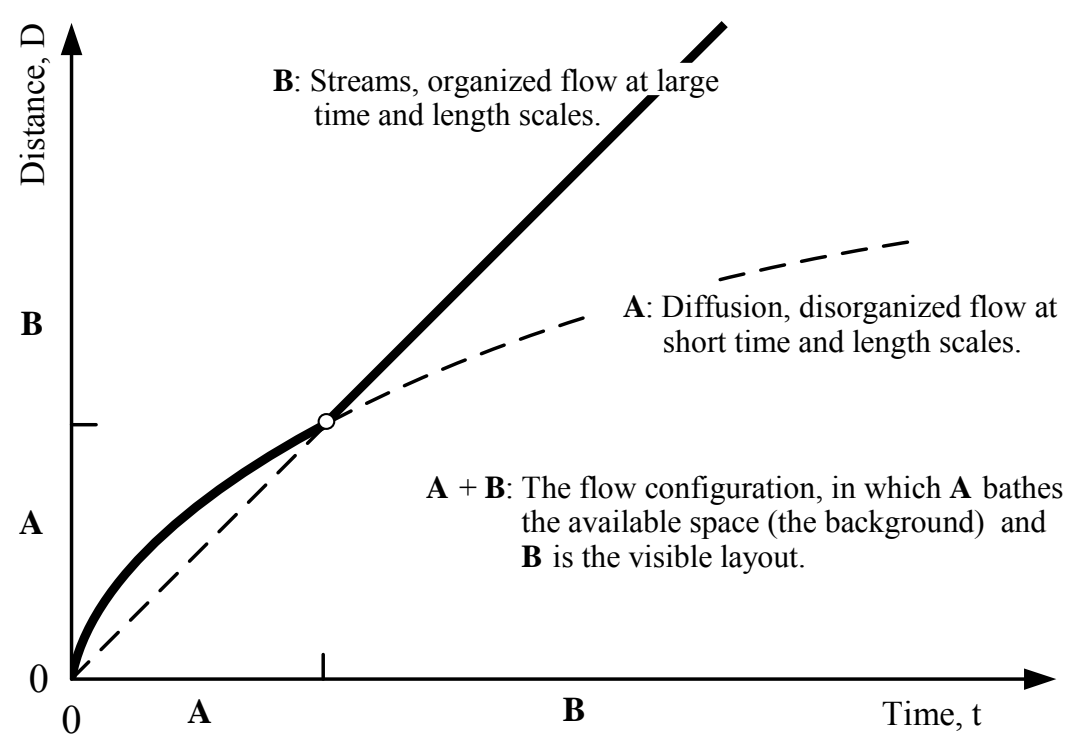

Figure 1 - Turbulence, dendritic solidification, electrodiffusion, mud cracks, dust aggregates, stony corals and bacterial colonies owe their configurations to the natural tendency to flow more easily. The distance traveled by diffusion increases in time as $t^{1 / 2}$, and the speed of the diffusion front decreases as $t^{-1 / 2}$. Greater flow access calls for a mechanism that is more effective at long times: convection (streams). Together, the two mechanisms provide greater flow access than one mechanism alone, provided that they are arranged in this order: diffusion at short time and length scales, woven with convection at larger scales. Nature always chooses this arrangement, not the opposite. 
momentum transport across $\mathrm{D}$ is by viscous diffusion (laminar flow), then $\mathrm{D}$ grows as $(\mathrm{vt})^{1 / 2}$, where $v$ is the kinematic viscosity. If momentum is transported by streams (rolls), then $\mathrm{D}$ acquires the scale Vt. Maximum flow access means that laminar shear is preferred at short times, and eddies at longer times. The transition between the two is marked by the generation of configuration: the first eddy. The intersection of the $\mathrm{D}(\mathrm{t})$ curves for the laminar and eddy shear leads to the prediction that transition occurs when the local Reynolds number $\mathrm{VD} / \mathrm{v}$ is of order $10^{2}$, where $\mathrm{V}$ and $\mathrm{D}$ are the peripheral speed and size of the first eddy. This agrees with all observations of transition in jets, wakes, plumes, shear layers, and boundary layers in forced and natural convection [2].

Dendritic solidification has the same origin. In a motionless fluid medium at a temperature slightly below the solidification temperature, latent heat is released at the solidification site and flows into the subcooled medium $[2,12]$ How does nature facilitate the flow of heat? It does by placing thermal diffusion at short times and needles at longer times. Thermal diffusion spreads as a spherical wave of radius $(\alpha t)^{1 / 2}$, where $\alpha$ is the thermal diffusivity of the medium. Needles grow at constant speed parallel to themselves. Here we encounter again the two-mechanism balance, and from this follows a theoretical basis on which to expect "rapid solidification"-needles growing on a background swept by thermal diffusion.

The same scenario rules the formation of needles and dendritic aggregates of dust particles in air and filter surfaces [13]. Stony corals, bacterial colonies and plant roots favor a small-size ball shape in the beginning of their growth [14]. Later, corals opt for larger structures with branches that invade the water flow. Nature selects diffusion at short times, and ion convection at longer times. The collaboration of the two mechanisms toward greater access is shown in general terms in Fig. 1.

\section{Line-to-line trees: the origin of multiscale porous media}

Natural porous flow structures also exhibit multiple scales and nonuniform distribution of length scales through the available space. Can such heterogeneous flow structures be anticipated based on the same principle of maximization of flow access? In Ref. [15] we showed that the answer is yes. We explored the properties and performance of a novel dendritic flow architecture proposed in Figs. 2 and 3. The idea is to connect two parallel lines (or two parallel planes) with trees that alternate with upside down trees. The resulting dendritic pattern connects the bottom boundary of the flow domain with the top boundary.

This alternating sequence of point-to-line trees constitutes a vasculature between the two parallel boundaries of the designed porous body. The fluid flows in the same direction through all the trees, e.g., upward in Fig. 3. This type of vascularization (line-to-line trees) establishes a multiscale designed porous medium between the long parallel boundaries of the vascularized body.
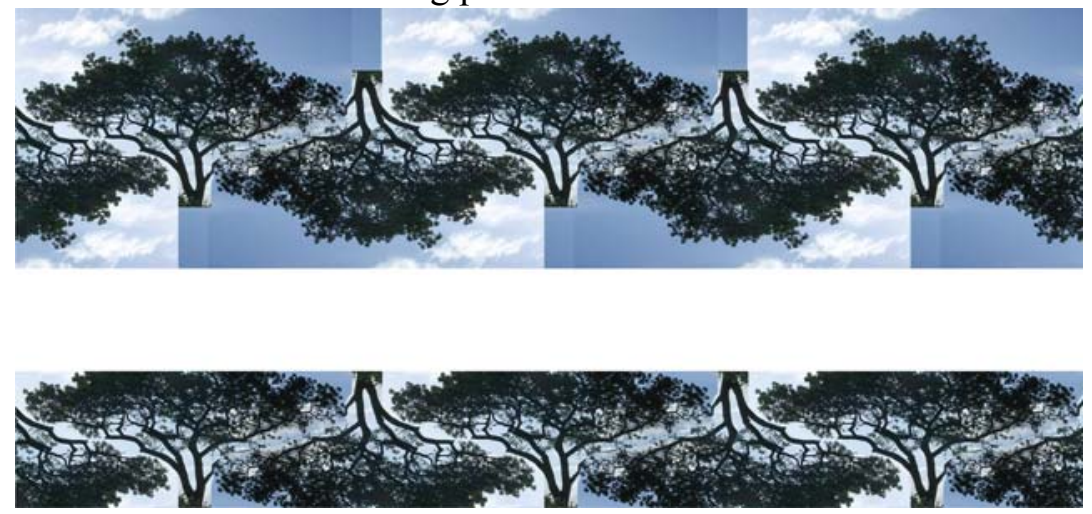

Figure 2 - Trees that alternate with upside down trees form a dendritic structure that connects one plane with another parallel plane [1]. 


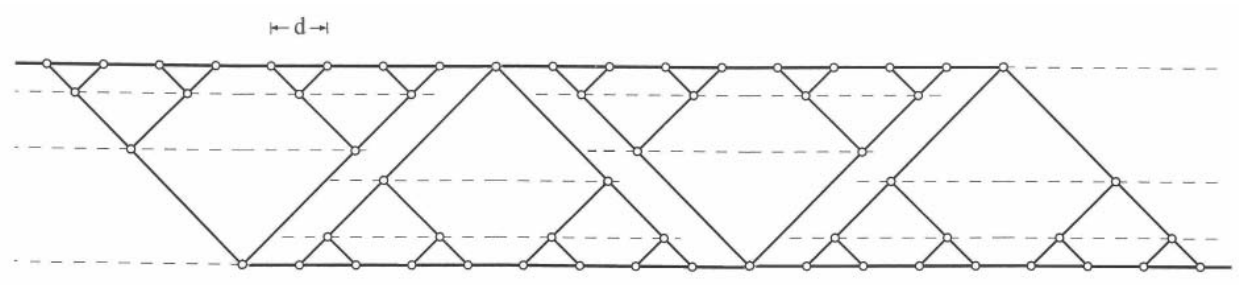

Figure 3 - Tree architecture for connecting one line with another line [15].

The maximization of flow access between the points of one line and the points of a parallel line can be viewed as a sequence of point-to-line flow access maximization problems. The building block with which Fig. 3 is constructed was proposed by Lorente et al. [16], where it was based on optimally shaped rectangular areas, each area allocated to one channel.

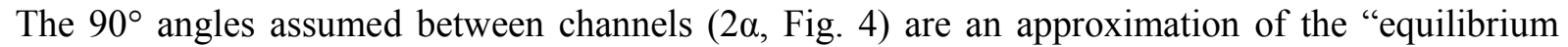
flow structure" [3] however, the flow performance of the approximate design comes close to the performance of the equilibrium structure.

Assume further that all the tubes are round and with Poiseuille flow, and that the svelteness number $\mathrm{Sv}$ is high such that pressure losses at the junctions can be neglected. Svelteness is a global flow property of the flow architecture [17],

$$
\mathrm{Sv}=\frac{\text { external flow length scale }}{\text { internal flow length scale }} \ldots
$$

When $\mathrm{Sv}>10$, the minimization of the pressure drop across the entire Y-shaped construct (subject to fixed total tube volume) yields the well known Hess-Murray law, according to which the ratio of successive tube diameters is $\mathrm{D}_{1} / \mathrm{D}_{2}=2^{1 / 3}$.

When there are many bifurcation levels $(i=1,2, \ldots, n)$, the tube lengths decrease by a factor of $1 / 2$, from the largest $\left(\mathrm{L}_{0}\right)$, to $\mathrm{L}_{1}=\mathrm{L}_{0} / 2, \mathrm{~L}_{2}=\mathrm{L}_{1} / 2$, etc. The smallest length scale is the smallest tube length,

$$
\mathrm{L}_{\mathrm{n}}=2^{-\mathrm{n}} \mathrm{L}_{0}
$$

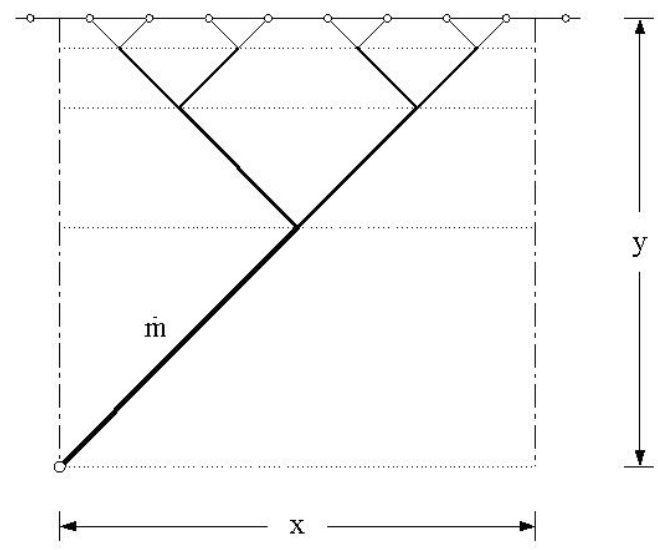

Figure 4 One of the point-to-line trees of Fig. 3 [15]. 
or the distance between the two ends of two neighboring $L_{n}$ tubes, $d=2 L_{n} \sin \alpha$. We carry $\alpha$ as a parameter, although in Fig. 4 the value is $\alpha=45^{\circ}$. The pressure drop along one tube of length $\mathrm{L}_{\mathrm{i}}$ and diameter $\mathrm{D}_{\mathrm{i}}$ is

$$
\Delta \mathrm{P}_{\mathrm{i}}=\dot{\mathrm{m}}_{\mathrm{i}} \frac{8}{\pi} \mathrm{Pov} \frac{\mathrm{L}_{\mathrm{i}}}{\mathrm{D}_{\mathrm{i}}^{4}}
$$

where Po is the Poiseuille constant (e.g., Po $=16$ for round tubes), which appears in the formula for the friction factor, $\mathrm{f}_{\mathrm{i}}=\mathrm{Po}_{\mathrm{N}} / \mathrm{Re}_{\mathrm{D}_{\mathrm{i}}}$, and $\mathrm{Re}_{\mathrm{D}_{\mathrm{i}}}-\mathrm{U}_{\mathrm{i}} \mathrm{D}_{\mathrm{i}} / v$ with $\mathrm{U}_{\mathrm{i}}=\dot{\mathrm{m}}_{\mathrm{i}} /\left(\rho \pi \mathrm{D}_{\mathrm{i}}^{2} / 4\right)$. Mass conservation at every junction requires that $\dot{\mathrm{m}}_{\mathrm{i}}=2 \dot{\mathrm{m}}_{\mathrm{i}+1}$, where it is again assumed that the tubes are sufficiently slender so that the asymmetry of the $\mathrm{Y}$ junction does not affect the splitting of $\dot{\mathrm{m}}_{\mathrm{i}}$ into two equal streams $\dot{m}_{\mathrm{i}+1}$. After using the ratios for diameters, lengths and mass flow rates indicated above, the total pressure drop from the open end of the $\mathrm{L}_{0}$ tube to the open ends of the $\mathrm{L}_{\mathrm{n}}$ tubes, becomes

$$
\Delta \mathrm{P}=\sum_{\mathrm{i}=1}^{\mathrm{n}} \Delta \mathrm{P}_{\mathrm{i}}=\dot{\mathrm{m}}_{0} \frac{8}{\pi} v \text { Po } \frac{\mathrm{L}_{0}}{\mathrm{D}_{0}^{4}} \sigma_{1}
$$

where $\sigma_{1}=1+2^{-2 / 3}+\ldots+2^{-2 n / 3}=\left[1-\left(2^{-2 / 3}\right)^{n+1}\right] /\left(1-2^{-2 / 3}\right)$ The total tube volume occupied by the tree flow is

$$
\mathrm{V}=\frac{\pi}{4}\left(\mathrm{D}_{0}^{2} \mathrm{~L}_{0}+2 \mathrm{D}_{1}^{2} \mathrm{~L}_{1}+\ldots+2^{\mathrm{n}} \mathrm{D}_{\mathrm{n}}^{2} \mathrm{~L}_{\mathrm{n}}\right)=\frac{\pi}{4} \mathrm{D}_{0}^{2} \mathrm{~L}_{0} \sigma_{1}
$$

The largest length scale $\left(\mathrm{L}_{0}\right)$ is related to the vertical dimension of the tree $(\mathrm{y})$ by

$$
\mathrm{y}=\left(\mathrm{L}_{0}+\mathrm{L}_{1}+\ldots+\mathrm{L}_{\mathrm{n}}\right) \cos \alpha=\mathrm{L}_{0} \sigma_{2} \cos \alpha
$$

where $\sigma_{2}=2\left[1-2^{-(\mathrm{n}+1)}\right]$. The horizontal dimension $(\mathrm{x})$ of the area occupied by the tree projection is

$$
\mathrm{x}=2^{\mathrm{n}} \mathrm{d}=2 \mathrm{~L}_{0} \sin \alpha=\frac{2}{\mathrm{~S}_{2}} \mathrm{y} \tan \alpha
$$

where $2^{n}$ is the number of $L_{n}$ tubes that reach the upper end of the construct. Eliminating $L_{0}$ and $D_{0}$ between Eqs. (4) - (6) we obtain

$$
\Delta \mathrm{P}=\dot{\mathrm{m}}_{0} \frac{\pi}{2} \mathrm{Po} \frac{\mathrm{v}}{\mathrm{V}^{2}}\left(\frac{\sigma_{1} \mathrm{y}}{\sigma_{2} \cos \alpha}\right)^{3}
$$

We question how effective the tree structure of Fig. 3 is relative to a well-known reference architecture: an array of $\mathrm{N}$ equidistant parallel tubes, each of length $\mathrm{y}$ and diameter $\mathrm{D}$. This classical structure carries the same total flow rate $\dot{\mathrm{m}}_{0}$ in the same total tube volume $\left(\mathrm{V}=\mathrm{N} \frac{\pi}{4} \mathrm{D}^{2} \mathrm{y}\right.$ ) and over the same area $x y / 2$. The structure has one degree of freedom, the tube diameter $\mathrm{D}$, or the number of parallel tubes, 


$$
\mathrm{N}=\frac{4 \mathrm{~V}}{\pi \mathrm{D}^{2} \mathrm{y}}
$$

The pressure drop along this structure $\left(\Delta \mathrm{P}_{\text {ref }}\right)$ is the same as the pressure drop along a single tube, cf. Eq. (3), through which the flow rate now is $\dot{\mathrm{m}}_{0} / \mathrm{N}$,

$$
\Delta \mathrm{P}_{\text {ref }}=\frac{\dot{\mathrm{m}}_{0}}{\mathrm{~N}} \frac{8}{\pi} \operatorname{Po} v \frac{\mathrm{y}}{\mathrm{D}^{4}}
$$

Eliminating D by using Eq. (9) we obtain

$$
\Delta \mathrm{P}_{\mathrm{ref}}=\dot{\mathrm{m}}_{0} \frac{\pi}{2} \mathrm{Po} \frac{v}{\mathrm{~V}^{2}} \mathrm{Ny}^{3}
$$

The tree-shaped structure of Fig. 3 has a smaller flow resistance than the parallel channels when $\Delta \mathrm{P}$ $<\Delta \mathrm{P}_{\text {ref, }}$ or, using Eqs. (8) and (11), when

$$
\mathrm{N}>\left(\frac{\sigma_{1}}{\sigma_{2} \cos \alpha}\right)^{3}
$$

The right side of this inequality is a number on the order of 1 . In conclusion, as the reference structure becomes finer (i.e., as $\mathrm{N}$ increases), the tree-shaped design of Fig. 3. becomes more attractive.

This conclusion can be read as a statement of how fine the tree structure must be such that it is preferable to the reference design. For a more practical comparison, assume that the smallest dimension that can be manufactured (d) is the same in both architectures, i.e. the d spacing of Fig. 3 is the same as the spacing between parallel tubes. This means that the number of parallel channels that occupy the area $\mathrm{y} \times(\mathrm{x} / 2)$ is $\mathrm{N}=2^{\mathrm{n}} / 2$, and when $\alpha=45^{\circ}$ the inequality (12) becomes

$$
\frac{\Delta \mathrm{P}}{\Delta \mathrm{P}_{\text {ref }}} \cong \frac{14}{2^{\mathrm{n}}}<1
$$

When the number of branching levels is 4 or larger, the tree-shaped architecture offers greater access to the flow that permeates through the porous structure of thickness $\mathrm{y}$. The superiority of the tree design increases fast as $\mathrm{n}$ increases: when $\mathrm{n}=7$, the ratio $\Delta \mathrm{P} / \Delta \mathrm{P}_{\text {ref }}$ is as low as $1 / 10$.

In conclusion, greater flow access means that when the available flow scales are sufficiently small (d), the flow architecture should be tree shaped, not parallel channels (single scale). From this follows the natural multiscale characteristic of porous media: large numbers of small pores and few large pores (known as "pipes" in hill slope hydrology).

\section{Vascular designs at large scales}

Society with all its layers and features of organization is a flow system. It is a "live" system, perhaps the most complex and puzzling we know. It is the most difficult to comprehend because we, the individuals who try to make sense of it, are inside the flow system. Each of us is like an alveolus in the lung, an eddy in a turbulent river, or a leaf on a tree branch. From such a position of singularity, which is identical in rank to the positions of enormous numbers of individuals, it is a formidable task to see and describe the big picture - the lung, the river basin, and the forest. 
The constructal law statement is general: it does not use words such as tree, complex versus simple, and natural versus engineered. The procedure used to deduce a class of flow configurations by invoking the constructal law is an entirely different (separate, subsequent) thought, which should not be confused with the constructal law. There are several (not many) classes of flow configurations, and each class can be derived from the constructal law in several ways, analytically (pencil \& paper) or numerically, approximately or more accurately, blindly (via random search) or using intelligence (strategy, short cuts), and so on. Classes that we have treated in detail, and by several methods, are the cross-sectional shapes of ducts, the cross-sectional shapes of rivers, internal spacings, and tree-shaped architectures $[2,7,11]$.

Regarding trees, our group treated them not as models (many have published and continue to publish models), but as fundamental access-maximization problems: volume to point, area to point, line to point, and the respective reverse flow directions. Important is the geometric notion that the "volume", the "area" and the "line" represent infinities of points. Our theoretical discovery of trees stems from the decision to connect one point (source, or sink) with an infinity of points (volume, area, line). The reality of the continuum is routinely approximated as a finite number of discrete points, which are then connected with 'sticks', which (of course) cover the space incompletely (and, from this, fractal geometry). Recognition of the continuum requires a study of the interstitial spaces between the tree links. The interstices can only be bathed by high-resistivity diffusion (an invisible, disorganized flow), while the tree links serve as conduits for low-resistivity organized flow (visible streams, ducts, channels).

The two modes of flowing with thermodynamic imperfection (i.e., with resistances), the interstices and the links, must be balanced so that together they contribute minimum imperfection to the global flow architecture. Choke points must be balanced and distributed. The flow architecture is the graphical expression of the balance between links and their interstices. The deduced (tree, duct shape, spacing, etc.) represents the optimal distribution of imperfection [7]. Modeling the natural trees by drawing them as black lines on white paper (while not optimizing the layout of every black line on its optimally sized and allocated white patch) leads to an incomplete drawing. The white is as important as the black.

Here we illustrate the optimal distributing of imperfection with two examples from our work on large-scale tree shaped networks. The first addresses the question of why streets are usually arranged in clusters (patterns, grids) that look almost similar from street to street and from city to

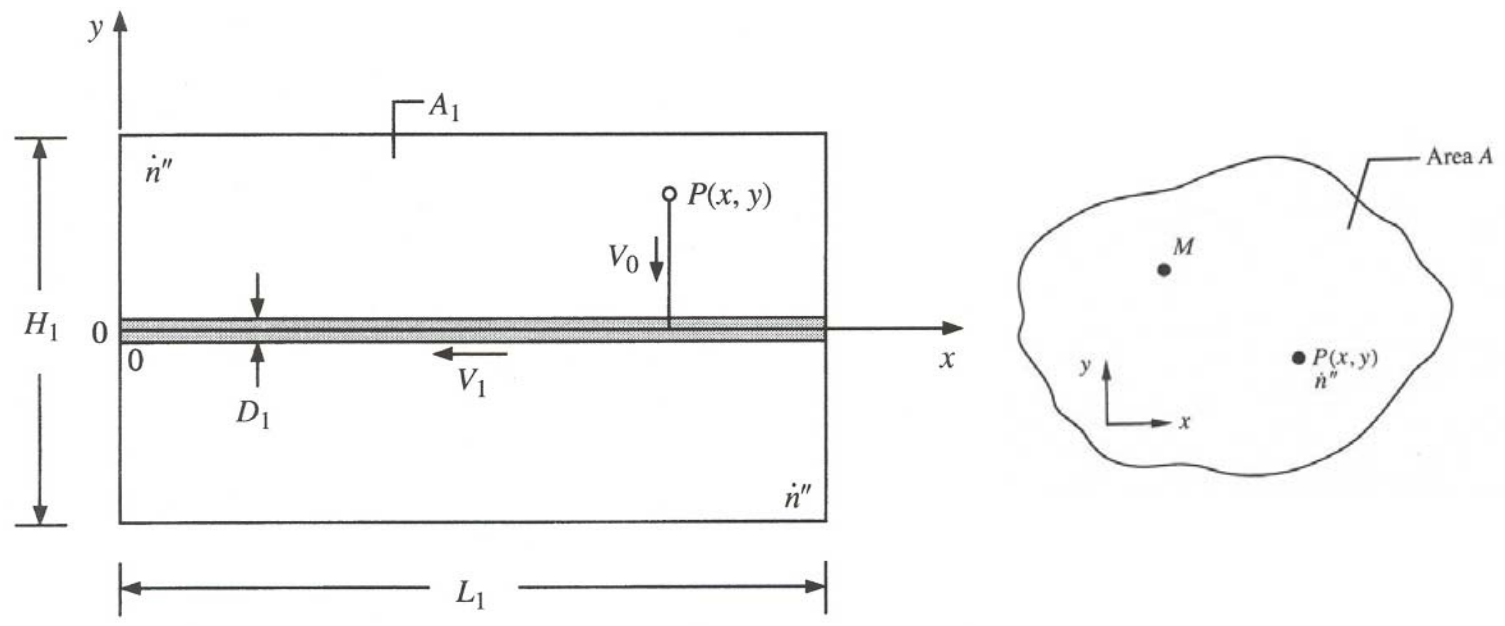

Figure 5 - Finite-size area (A) covered by a uniformly distributed population $\left(\dot{\mathrm{n}}^{\prime \prime}\right)$ traveling to a common destination (M), and the street allocated to the smallest (innermost) elemental area [2]. 
city [2]. Indeed, why do streets exist? Constructal theory provided answers to these questions by formulating the following area-point access maximization problem.

Consider a finite-size geographical area $\mathrm{A}$ and a point $\mathrm{M}$ situated inside $\mathrm{A}$ or on its boundary (Fig. 5). Each member of the population living on A must travel between his or her point of residence $\mathrm{P}(\mathrm{x}, \mathrm{y})$ and the point $\mathrm{M}$. The latter serves as common destination for all the people who live on A. The density of this traveling population-i.e., the rate at which people must travel to $\mathrm{M}$ - is fixed and described by $\dot{\mathrm{n}}^{\prime \prime}$ (people $/ \mathrm{m}^{2} \mathrm{~s}$ ). This also means that the rate at which people are streaming into $\mathrm{M}$ is constrained, $\dot{\mathrm{n}}=\dot{\mathrm{n}}^{\prime \prime} \mathrm{A}$. Determine the optimal bouquet of paths that link the points $\mathrm{P}$ of area $\mathrm{A}$ with the common destination $\mathrm{M}$ such that the time of travel required by the entire population is the shortest.

The problem is how to connect a finite area (A) to a single point (M). Area A contains an infinite number of points, and every one of these points must be taken into account when optimizing the access from $\mathrm{A}$ to $\mathrm{M}$, and from $\mathrm{M}$ to $\mathrm{A}$.

Our first analytical approach to this problem was "atomistic", from the smaller subsystem (detail) of area A, to the larger subsystem, and ultimately to area A itself. The area subsystem to which a street length may be allocated cannot be smaller than the size fixed by the living conditions (e.g., property) of the people who will be using the street. This smallest area scale is labeled $A_{1}$ in Fig. 5 . Although $A_{1}$ is fixed, its shape or aspect ratio $H_{1} / L_{1}$ is not.

Symmetry suggests that the best position for the street segment is along the longer of the axes of symmetry of $A_{1}$. This choice has been made in Fig. 5, where $L_{1}>H_{1}$ and the street has the length $\mathrm{L}_{1}$ and width $\mathrm{D}_{1}$. The traveling population density $\dot{\mathrm{n}}^{\prime \prime}$ is distributed uniformly on $\mathrm{A}_{1}$. To get out of $A_{1}$, each person must travel from a point of residence $\mathrm{P}(\mathrm{x}, \mathrm{y})$ to the $(0.0)$ end of the street. The person can travel at two speeds: (1) a low speed $\mathrm{V}_{0}$ when off the street, and (2) a higher speed $\mathrm{V}_{1}$ when on the street. See also Section 2.

The optimal shape $\mathrm{H}_{1} / \mathrm{L}_{1}$ was determined in two ways, with the same result. First, the travel time from any point $P$ to $(0,0)$ was averaged over $A_{1}$, and minimized with respect to $H_{1} / L_{1}$. Second, the travel time from the farthest corner $\left(\mathrm{L}_{1}, \mathrm{H}_{1} / 2\right)$ was minimized with respect to $\mathrm{H}_{1} / \mathrm{L}_{1}$. The result

$$
\left(\frac{\mathrm{H}_{1}}{\mathrm{~L}_{1}}\right)_{\mathrm{opt}}=\frac{2 \mathrm{~V}_{0}}{\mathrm{~V}_{1}}<1
$$

shows that the smallest rectangular area should be slender when the street velocity is sensibly greater than the lowest (walking) velocity. The rectangular area $\mathrm{A}_{1}$ must become more slender as $\mathrm{V}_{1}$ increases relative to $\mathrm{V}_{0}$ - that is, as time passes and technology advances. This trend is confirmed by a comparison between the streets built in antiquity and those that are being built today. In antiquity the first streets were short, typically with two or three houses on one side. In the housing developments that are being built today, the first streets are sensibly longer, with 10 or more houses on one side.

That Eq. (14) is obtained in two ways is important. It is important that the optimization of the shape of the $\mathrm{A}_{1}$ element is of interest to every inhabitant: What is good for the most disadvantaged person is good for every member of the community. This conclusion has profound implications in the spatial organization of all living groups, from bacterial colonies all the way to our own societies. The urge to organize is an expression of selfish behavior.

Important is also the discovery that when $\mathrm{H}_{1} / \mathrm{L}_{1}$ is optimal, the walking time $t_{0}=\left(\mathrm{H}_{1} / 2\right) / \mathrm{V}_{0}$ is equal $1^{\text {st }}$ International Workshop "Shape and Thermodynamics" - Florence 25 and 26 September 2008 
to the riding time $t_{1}=L_{1} / V_{1}$. The balancing of the flow times is another way to describe the configuration. The time to move slowly over a short distance is the same as the time to move fast over a long distance. This design feature is prevalent in natural flow systems and social dynamics. One example is the shape of the Atlanta airport, where the time to walk down half a concourse is the same as the time to ride on the train perpendicular to the six concourses.

The area elements (e.g. $A_{1}$, Fig. 5) are assembled and linked on a larger territory by streets that are progressively wider and faster. This construction is described in Refs. [2, 7]. The net result is that if the area A has only one point of interest M (Fig. 5), then the flow from A to M (or from M to A) is tree shaped. The urban design of an area has a large number of points of interest $\left(\mathrm{M}_{1}, \mathrm{M}_{2}, \ldots\right)$, and each flow $\left(\mathrm{A}-\mathrm{M}_{1}, \mathrm{~A}-\mathrm{M}_{2}, \ldots\right)$ is tree shaped. All these trees must coexist when projected on $\mathrm{A}$, and the configuration of streets that accommodates all flows is the grid. The actual flows are tree-shaped, not grid-shaped. In other words, the grid-shaped infrastructure is deceiving: the grid is a superposition of tree-shaped flows, and it is necessary because trees are necessary.

Tree-shaped flows are the vasculature of urban design, and they carry a diversity of streams: traffic, people, goods, sewage, water runoff, mail, information, etc. Urban design means flow (life) and the performance of its flow structures is robust. Here we illustrate the robustness with an example from the distributing of hot water over an area $[18,19]$.

The distribution of hot water to users on a specified territory presents two problems to the thermal designer: the fluid mechanics problem of minimizing the pumping power, and the heat transfer problem of minimizing the loss of heat from the piping network. The water flow is from one point (the source) to an area-the large number of users spread uniformly over the area.

The area $A$ is supplied with hot water by a stream of flow rate $\dot{m}$ and initial temperature $T_{i}$. The stream enters the area from the outside, by crossing one of its boundaries. The area is inhabited by a large number of users, $\mathrm{n}=\mathrm{A} / \mathrm{A}_{0}$, where $\mathrm{A}_{0}$ is the area element allocated to a single user, for example, the square $\mathrm{L}_{0} \times \mathrm{L}_{0}$ shown in Fig. 6. Each $\mathrm{A}_{0}$ element must receive an equal share of the original stream of hot water, $\dot{\mathrm{m}}_{0}=\dot{\mathrm{m}}_{\mathrm{n}}$. The ambient temperature is $\mathrm{T}_{\infty}$, where $\mathrm{T}_{\infty}<\mathrm{T}_{\mathrm{i}}$. The water pipes lose heat to the ambient, and to reduce this loss all the pipes are insulated with wrappings of insulation of thermal conductivity $\mathrm{k}$. The total volume of insulation material (V) is fixed and serves as constraint. The total flow volume of all the pipes is also fixed.

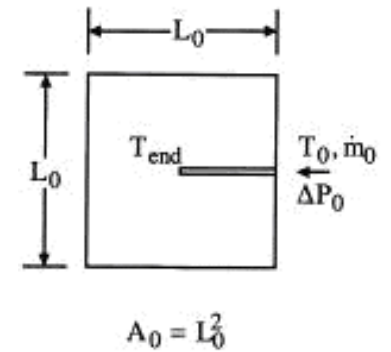

(0) Elemental System

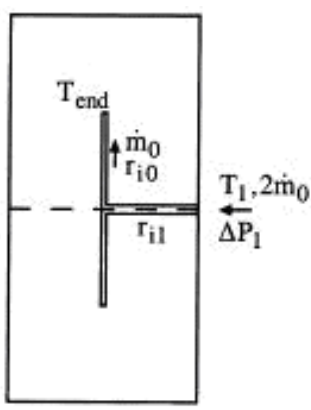

$$
\mathrm{A}_{1}=2 \mathrm{~L}_{0}^{2}
$$

(1) First Construct

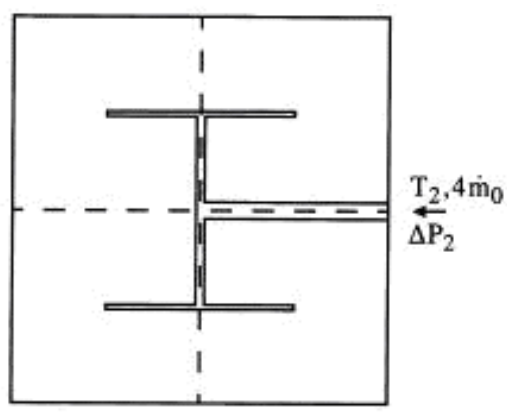

$$
\mathrm{A}_{2}=2^{2} \mathrm{~L}_{0}^{2}
$$

(2) Second Construct

Figure 6-Dichotomous networks of pipes with hot water: sequence of area constructs obtained by pairing smaller constructs [18]. 


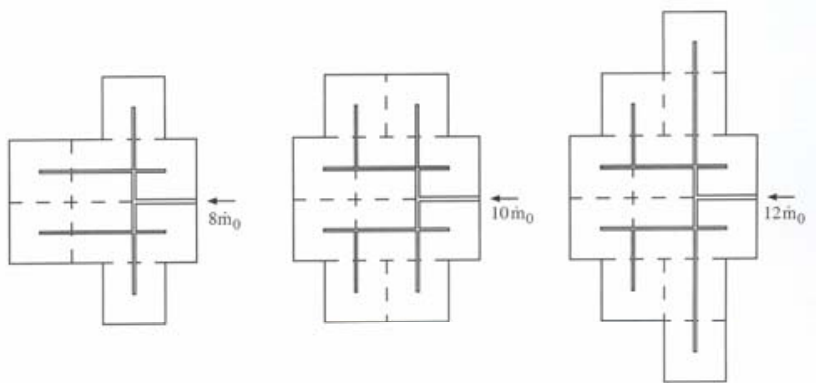

(a)

(b)

(c)

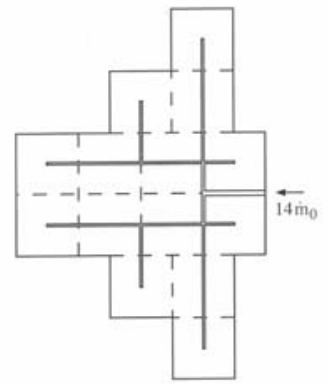

(d)

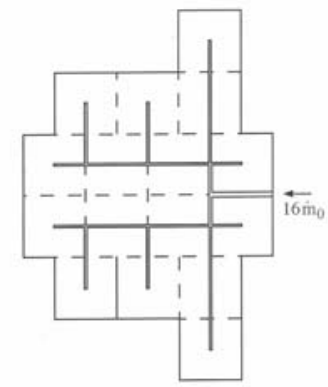

(c)

Figure 7 - Tree-shaped distribution of hot water where each new user $\left(A_{0}\right)$ is attached to the best branch of the preceding assembly [19].

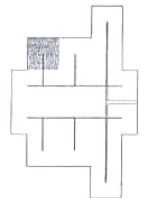

Fig. $7 \mathrm{e}$

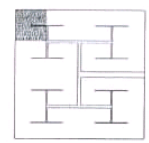

A4, Fig. 6

$\frac{\left(\mathrm{T}_{16}-\mathrm{T}_{\infty}\right)_{\text {Fig. } 7 \mathrm{e}}}{\left(\mathrm{T}_{\text {end }}-\mathrm{T}_{\infty}\right)_{\mathrm{A}_{4} \text { Fig. } 6}}$

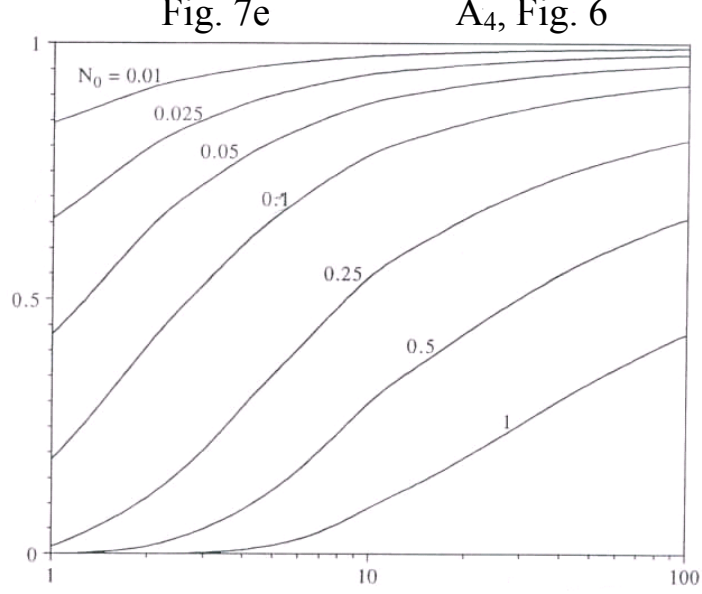

Figure 8 - Comparison between the excess temperature $\tilde{\mathrm{V}}$ of the water received by the last user added to the structure of Fig. 3e and the corresponding temperature in the design based on the sequence of Fig. 6. [19]. 
The search for the flow architecture is guided by two objectives simultaneously: (i) minimum global flow resistance, or pumping power, and (ii) minimum total loss of heat from network to ambient. Fundamental features that we discovered in this manner are the ratios of successive pipe diameters, and the radii of the insulation sleeve to be installed on every pipe. We also found that dichotomous tree-shaped structures (Fig. 6) are better than other tree constructions. In Fig. 8 we illustrate the design quality of robustness, which becomes more accentuated as the network covers a larger area. Compared are two designs of the same size, $\mathrm{A}=16 \mathrm{~A}_{0}$. The abscissa indicates the dimensionless insulation volume, defined in Ref. [18]. The number $\mathrm{N}_{0}$ is the number of heat loss units, defined as $\mathrm{N}_{0}=\pi \mathrm{kL}_{0} /\left(\dot{\mathrm{m}}_{0} \mathrm{c}_{\mathrm{p}}\right)$. The figure shows that when the insulation is of high quality (low $\mathrm{N}_{0}$ ) and sufficiently abundant (high $\tilde{\mathrm{V}}$ ) the temperature of the water stream $\left(\mathrm{T}_{16}\right)$ received by the farthest user approaches the corresponding temperature in the reference (best) design, Fig. 6. Noteworthy is that in Fig. 6 all the elemental areas receive their hot water at the same temperature $\left(\mathrm{T}_{\mathrm{end}}\right)$, i.e. the dichotomous network distributes the hot water uniformly. We repeated the comparison of Fig. 8 by varying the size of the flow system (e.g., $\mathrm{A}=8 \mathrm{~L}_{0}^{2}$ ), and demonstrated that the performance of Fig. 3e approaches more closely the performance of Fig. 6 as the size and complexity of the design increase [19].

\section{Multi-objective flow configurations}

The presence of two or more simultaneous objectives is emerging as the driving force in the generation of many constructal designs. Multiple objectives is the paradigm in animal design, where there is less controversy about how one objective is pursued through design (e.g., minimization of food use in flight [7]) than the length of the list of objectives and mechanisms that may be in play [10].

One class of two-objective architectures is appearing now frequently in the literature: tree-shaped architectures for heat exchangers, electronics cooling, smart materials, and high-density volumetric transport in general [20-25]. Figure 9 shows how the evolution of configuration drives the twoobjective performance in the natural direction. In heat transfer structures with convection, the two objectives are low global thermal resistance (heat transfer imperfection) and low fluid-flow resistance or pumping power (fluid-flow imperfection). A given flow architecture is represented by a fixed curve in Fig. 9. The curve is traced in the two-dimensional field as the mass flow rate varies: the mass flow rate varies along the curve from the upper left toward the lower right.

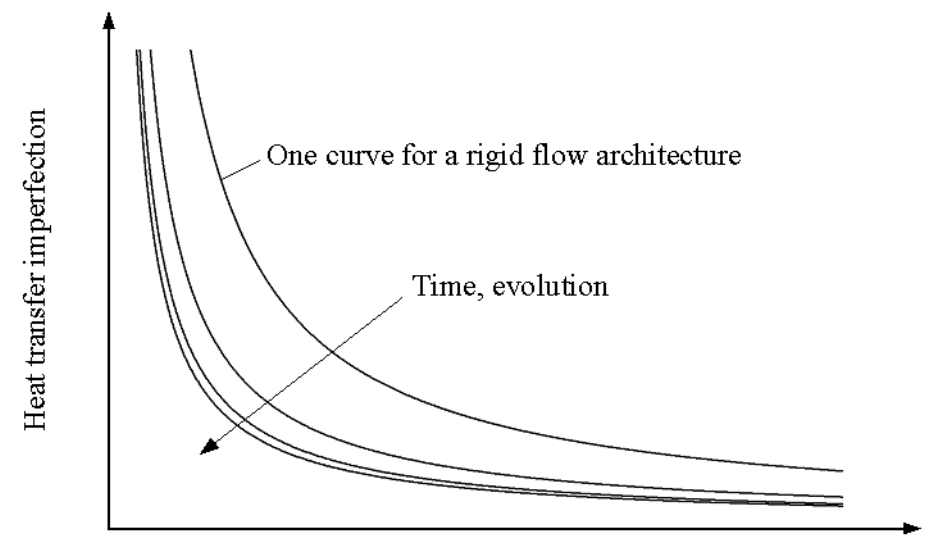

Fluid-flow imperfection

Figure 9 - The time evolution of flow configurations with two objectives: low global thermal resistance and low pumping power [1]. 
Better flow architectures are represented by curves that lie closer to the origin. Curves do not migrate: existing curves disappear and new curves appear, as the existing designs are discarded and replaced with new designs. When the new design is dramatically different than the classical design, the new curve executes a finite step in the evolutionary direction. Such steps are being registered now with dendritic heat exchanger structures relative to classical structures with parallel channels [15], cf. Section 3.

The evolutionary process suggested by Fig. 9 is the heart of the evolution of technology and science $[26,27]$. In the new book [1] we showed that the step changes (the transitions) exhibited by cooling technology are in line with the time arrow of Fig. 9. Examples are the jump from natural convection to forced convection for the volumetric cooling of electronics (Ref. [1], Section 3.7), and more recently, the jump from forced convection to solid-body conduction (Ref. [1], Section 5.4).

Our own work of applying constructal theory to two-objective designs began with an opportunity in the design of energy efficient buildings [24]. The wall of a heated room is a better insulator when air spaces (caverns) are placed inside the wall. More and larger caverns mean better insulation, but they also mean lower mechanical strength. We showed that when the wall must have simultaneously high thermal resistance and high strength, it is possible to identify the right number and thicknesses of the caverns that should be made in the wall. The optimal caverns become narrower and more numerous when the natural convection effect is stronger, i.e. when the wall is taller and the temperature difference across it is larger.

The challenge to combat terrorism brought another two-objective design to the forefront: structures that are mechanically strong and, at the same time, must be resistant to sudden and intense heating [25]. The principle is illustrated in Fig. 10 with a simply supported beam loaded vertically with uniform weight per unit length. The amount of beam material is fixed, but the shape of the beam profile may vary. The beam thickness (measured vertically) varies as $\mathrm{H}=\mathrm{C}[\mathrm{x}(\mathrm{L}-\mathrm{x})]^{\mathrm{m}}$, where $\mathrm{x}=$ 0 and $x=L$ represent the two ends, and the constant $C$ is dictated by the beam volume constraint. The shape of the beam profile is indicated by the exponent $\mathrm{m}$.

We found that there is an optimal shape $(\mathrm{m})$ such that the beam is most resistant mechanically and thermally. When the beam is exposed to intense heating, regions of thermoplasticity eat into the elastic body of the beam: the initial elastic thickness $\mathrm{H}(\mathrm{x})$ is reduced to an elastic core of thickness

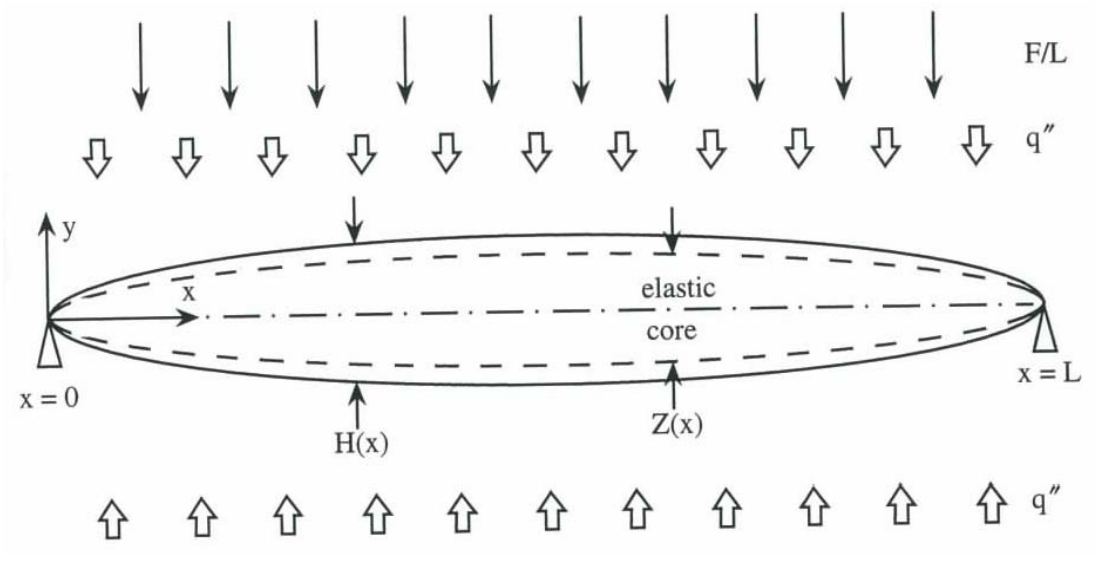

Figure 10 - Elastic beam in bending, loaded uniformly and exposed suddenly to intense heating from above and below [25]. 
$\mathrm{Z}(\mathrm{x})$, which decreases in time. The global strength (stiffness) of the beam decreases in time. The challenge is to shape the beam such that the degradation of its mechanical strength is slowed down the most. The solution is to put more material to act as "thermal insulation" at locations (x) where the bending moment is greater, i.e. where mechanical strength is needed most. The design evolves qualitatively toward the shape sketched in Fig. 10, with a shape exponent $\mathrm{m}$ in the range $0.5-0.56$.

The same principle leads to the optimal placement of reinforcing steel bars inside beams of concrete subjected to bending. From the point of view of increasing mechanical strength, the steel bars must be embedded near the bottom surface of the beam. For greater resistance to intense heating, the steel bars must be embedded in the center of the concrete. The two demands compete and generate an optimal position for the steel bars, i.e. the optimal configuration of the cross-section of the composite material. Details of this application of constructal design are available in Refs. [1, 25].

\section{Scaling up}

The constructal work on vascular design at small and large scales is a promising direction to solving the toughest of all problems in engineering design: scaling, i.e., how to use the results from a desksize model in order to predict the behavior and performance of "the same" system but at much larger scales. The difficulty stems from the nature of all flow systems: the larger is not "the same" as the laboratory model. The larger is not a magnified replica of the model.

What happens during the magnification exercise is suggested in Fig. 7 and the recent deduction that vascular designs must emerge, persist and become more complex if the flow system grows [27]. The configuration changes, because the flow system must be the best that it can be at any size. One cannot predict the performance of a large flow system by extrapolating from the tested performance of small model.

The only way to crack the scaling nut is to have a firm grip on the hammer of principles, i.e., to know how the system configuration changes as its size increases. Once we know the drawing, large or small, we can analyze (or test) the flow system and describe its performance with confidence. This means that if we know that Fig. 7e will be the configuration at large scales, then we must test in the laboratory a miniature of Fig. 7e, not of Fig. 7a. This knowledge is a powerful new tool, and a very timely one for placing the subject of "design" on a scientific basis [1].

\section{References}

1] A. Bejan and S. Lorente, Design with Constructal Theory, Wiley, Hoboken, 2008.

2] A. Bejan, Advanced Engineering Thermodynamics, $2^{\text {nd }}$ edn, Wiley, New York, 1997.

3] A. Bejan and S. Lorente, Constructal theory of generation of configuration in nature and engineering, J. Appl. Phys. 100, 041301 (2006).

4] H. Poirier, Une théorie explique l'intelligence de la nature, Science \& Vie, No. 1034 2003, pp. 44-63.

5] A. H. Reis, Constructal theory: from engineering to physics, and how systems flow develop shape and structure, Applied Mechanics Reviews Vol. 59, 2006, pp. 269-281.

6] A. Bejan and G. W. Merkx, eds., Constructal Theory of Social Dynamics, Springer, New York, 2007.

7] A. Bejan, Shape and Structure, from Engineering to Nature, Cambridge University Press, Cambridge, UK, 2000.

8] A. Bejan and J. H. Marden, Unifying constructal theory for scale effects in running, swimming and flying, J. Exp. Biol., Vol. 209, 2006, pp. 238-248.

9] A. Bejan and J. H. Marden, Constructing animal locomotion from new thermodynamics theory, American Scientist, July-August 2006, pp. 343-349.

10] H. Hoppeler and E. R. Weibel, eds., Scaling Functions to Body Size: Theories and Facts, 
special issue, J. Exp. Biol., Vol. 208(9), 2005.

11] A. Bejan, Advanced Engineering Thermodynamics, $3^{\text {rd }}$ eds., Wiley, Hoboken, 2006.

12] A. Ciobanas, A. Bejan and Y. Fautrelle, Dendritic solidification morphology viewed from the perspective of constructal theory, J. Phys. D: Appl. Phys., Vol. 39, 2006, pp. 5252-5266.

13] A. H. Reis, A. F. Miguel and A. Bejan, Constructal theory of particle agglomeration, J. Phys. D: Appl. Phys., Vol. 39, 2006, pp. 2311-2318.

14] A. F. Miguel, Constructal pattern formation in stony corals, bacterial colonies and plant roots under different hydrodynamic conditions, J. Theoretical Biology, Vol. 242, 2006, pp. 954-961.

15] S. Lorente and A. Bejan, Heterogeneous porous media as multiscale structures for maximum flow access, J. Appl. Phys., Vol. 100, 2006, 114909.

16] S. Lorente, W. Wechsatol and A. Bejan, Tree-shaped flow structures designed by minimizing path lengths, Int. J. Heat Mass Transfer, Vol. 45, 2002, pp. 3299-3312.

17] S. Lorente and A. Bejan, Svelteness, freedom to morph, and constructal multi-scale flow structures, Int. J. Thermal Sciences, Vol. 44, 2005, pp. 1123-1130.

18] W. Wechsatol, S. Lorente, and A. Bejan, Tree-shaped insulated designs for the uniform distribution of hot water over an area, Int. J. Heat Mass Transfer, Vol. 44, 2001, pp. 31113123.

19] W. Wechsatol, S. Lorente, and A. Bejan, Development of tree-shaped flows by adding new users to existing networks of hot water pipes, Int. J. Heat Mass Transfer, Vol. 45, 2002, pp. 723-733.

20] S. Kim, S. Lorente and A. Bejan, Vascularized materials: tree-shaped flow architectures matched canopy to canopy, J. Appl. Phys., Vol. 100, 2006, 063525.

21] J. Lee, S. Kim, S. Lorente and A. Bejan, Vascularization with trees matched canopy to canopy: diagonal channels with multiple sizes, Int. J. Heat Mass Transfer, Vol. 51, 2008, pp. 2029-2040.

22] K.-M. Wang, S. Lorente and A. Bejan, Vascularized networks with two optimized channels sizes, Journal of Physics D: Applied Physics, Vol. 39, 2006, pp. 3086-3096.

23] W. Wechsatol, S. Lorente and A. Bejan, Dendritic convection on a disc, Int. J. Heat Mass Transfer, Vol. 46, 2003, pp. 4381-4391.

24] S. Lorente and A. Bejan, Combined 'flow and strength' geometric optimization: internal structure in a vertical insulating wall with air cavities and prescribed strength, Int. J. Heat Mass Transfer, Vol. 45, No. 16, 2002, pp. 3313-3320.

25] L. Gosselin, A. Bejan and S. Lorente, Combined 'heat flow and strength' optimization of geometry: mechanical structures most resistant to thermal attack", Int. J. Heat Mass Transfer, Vol. 47, 2004, pp. 3477-3489.

26] A. Bejan, Science and technology as evolving flow architectures, Int. J. Energy Research, Vol. 32, 2008, in press.

27] S. Kim, S. Lorente and A. Bejan, The emergence of vascular design in three dimensions, $J$. Appl. Phys., Vol. ?, 2008, to appear. 